\title{
Resolution in Plenoptic Cameras
}

Todor Georgiev

Adobe Systems, 345 Park Ave, San Jose, CA 95110

tgeorgie@adobe.com
Andrew Lumsdaine

Indiana Univ., 215 Lindley Hall, Bloomington, IN 47405

lums@osl.iu.edu

\begin{abstract}
Derivation and analysis of sampling patterns of traditional and focused plenoptic cameras show the former rotates pixels $\pi / 2$ in phase space, while the latter does not. These results are interpreted regarding the cameras' spatial resolution.
\end{abstract}

(C) 2009 Optical Society of America

OCIS codes: (080.2730) Matrix methods in paraxial optics, (040.1490) Cameras.

\section{Introduction}

The Plenoptic camera, a digital realization of Lippmann's integral photography ideas [4], was introduced in 1992 [1] as an approach to solve computer vision problems. In this camera, microlenses are placed one focal length from the sensor, and are thus focused at infinity. Each microlens focuses parallel rays coming to it from a given direction onto a single point on the sensor, thus converting angular distribution into spatial. As we show later in the paper, this conversion of angular into spatial information is related to rotation in optical phase space.

The Plenoptic 2.0 camera, also related to the ideas of Lippmann [4], has been independently introduced in [7, 2, 5] and others. In its current version, it has microlenses placed at distance $b$ from the sensor, so that they are focused at the image plane of the main camera lens, a distance $a$ in front of them. In this configuration, they satisfy the lens equation $1 / a+1 / b=1 / f$ and construct a relay system with the main lens. The fundamental difference between Plenoptic 2.0 and Plenoptic 1.0 is that in the former, the microimages are focused on the scene (through the relay system), while in the latter they are completely defocused relative to the scene. See Fig. 1.
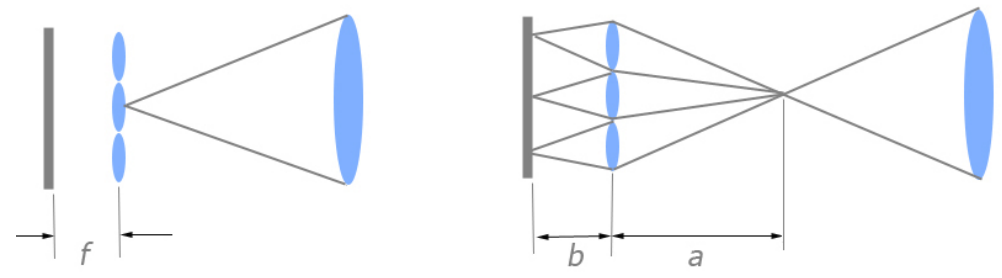

Fig. 1. Plenoptic 1.0 (left) and Plenoptic 2.0 (right) cameras.

A main practical consequence of the difference between the two plenoptic cameras is in the resolution of the final rendered image. While Plenoptic 1.0 produces one pixel per microlens in the final image, Plenoptic 2.0 produces multiple pixels. This decoupling between the number of microlenses and the number of pixels is a significant capability of the Plenoptic 2.0 camera [6].

To more fully and rigorously analyze the imaging capabilities of the two types of plenoptic camera, this paper derives the expressions for the spatioangular sampling performed by the sensor, which we show with phase space diagrams describing each camera. This derivation builds intuition about both cameras and explains the the spatioangular tradeoffs and higher resolution of Plenoptic 2.0 camera. 


\section{Transfer matrices in ray optics}

Refraction by a lens and travel of rays in free space is described by two matrix transforms, $L$ and $T$.

$$
L=\left[\begin{array}{cc}
1 & 0 \\
-\frac{1}{f} & 1
\end{array}\right], T=\left[\begin{array}{ll}
1 & t \\
0 & 1
\end{array}\right]
$$

These matrices act on rays, represented by $x=(q, p)^{T}$, where $q$ is the position, $p$ is the slope (angle) of the given ray relative to the optical axis.

In this notation, the lightfield [3] (or plenoptic function) is simply the radiance as a function in ray space, $r(x)$. An arbitrary ray transfer matrix $A$ transforms each ray according to $x^{\prime}=A x$, and it transforms the radiance $r(x)$ into $r^{\prime}(x)$. First we will derive the transformation formula

$$
r^{\prime}(x)=r\left(A^{-1} x\right)
$$

Since optical transfer matrices satisfy $\operatorname{det} A=1$, and assuming conservation of energy, we have $r^{\prime}\left(x^{\prime}\right)=r(x)$, or equivalently $r^{\prime}(A x)=r(x)$. Considering a ray $y=A x$, we thus obtain $r^{\prime}(y)=r\left(A^{-1} y\right)$. Since $y$ is an arbitrary ray, we obtain (2).

\section{Plenoptic 1.0}

Based on the camera design, it should be clear that radiance sampling in the Plenoptic 1.0 camera should be described by Fig. 2. Each pixel under a given microlens measures the energy coming to that microlens as rays at a certain angle.
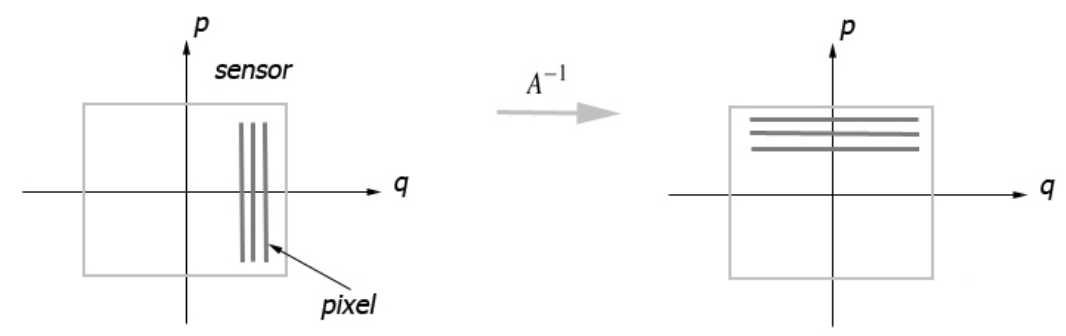

Fig. 2. Sampling pattern of one microlens in Plenoptic 1.0 camera.

To derive that relationship, we compute the matrix $A$ and $A^{-1}$ for rays incident to a given microlens.

$$
A=\left[\begin{array}{ll}
1 & f \\
0 & 1
\end{array}\right]\left[\begin{array}{cc}
1 & 0 \\
-\frac{1}{f} & 1
\end{array}\right]=\left[\begin{array}{cc}
0 & f \\
-\frac{1}{f} & 1
\end{array}\right], A^{-1}=\left[\begin{array}{cc}
1 & -f \\
\frac{1}{f} & 0
\end{array}\right]
$$

Consider equation (3). A pixel on the sensor responds approximately equally to all angles. Therefore its sampling kernel in ray space is represented as a vertical line as thick as the pixel in Fig. 2. $A^{-1}$ maps this vertical line to a horizontal line because due to the bottom right matrix element, input $p$ doesn't influence output $p$. This transform contains rotation and shearing. That is, pixels become horizontal. Moreover, their size (the amount sampled in the spatial domain) is limited by the microlens diameter.

The sampling characteristics of the Plenoptic 1.0 camera have ramifications for plenoptic camera design. In the sampling pattern of the Plenoptic 1.0 camera, the spatial resolution is equal to the size of a microlens and the angular resolution is equal to the number of pixels under a microlens. I.e., the spatio-angular tradeoff is completely determined by the number of microlenses. To get good spatial resolution in this case, we require a large number of small microlenses.

However, there is a limit to the effectiveness of a Plenoptic 1.0 camera with small microlenses. A small number of pixels per microlens produces noisy and unreliable results due to edge effects at each microimage. To counter edge effects, we need a fairly large number of pixels under each microlens (more than 100). Thus, the Plenoptic 1.0 design 
excludes certain regions of the spatio-angular tradeoff space. In particular, it excludes high spatial resolution and low angular resolution. Since Lambertian surfaces produce radiance that has redundancy in angle, high spatial sampling could be used because only sparse angular sampling would be required.

\section{Plenoptic 2.0}

The Plenoptic 2.0 camera differs from the previous one by recording radiance not at the microlenses, but at a plane located a distance $a$ in front of the microlenses. The total transfer matrix is

$$
A=\left[\begin{array}{ll}
1 & b \\
0 & 1
\end{array}\right]\left[\begin{array}{cc}
1 & 0 \\
-\frac{1}{f} & 1
\end{array}\right]\left[\begin{array}{ll}
1 & a \\
0 & 1
\end{array}\right]=\left[\begin{array}{cc}
-\frac{b}{a} & 0 \\
-\frac{1}{f} & -\frac{a}{b}
\end{array}\right] .
$$

The last equality holds due to focusing. Computing the inverse,

$$
A^{-1}=\left[\begin{array}{cc}
-\frac{a}{b} & 0 \\
\frac{1}{f} & -\frac{b}{a}
\end{array}\right]
$$

The important observation here is that due to the zero top right element, pixels are not rotated in optical phase space. A vertical line remains vertical after inverse mapping. As a result, sampling is done by a dense set of thin vertical kernels, and is decoupled from microlens size. See Fig. 3. Considering that minification for each microcamera is $a / b$, the high spatial resolution achieved is $b / a$ times the sensor resolution. See Fig. 3 .
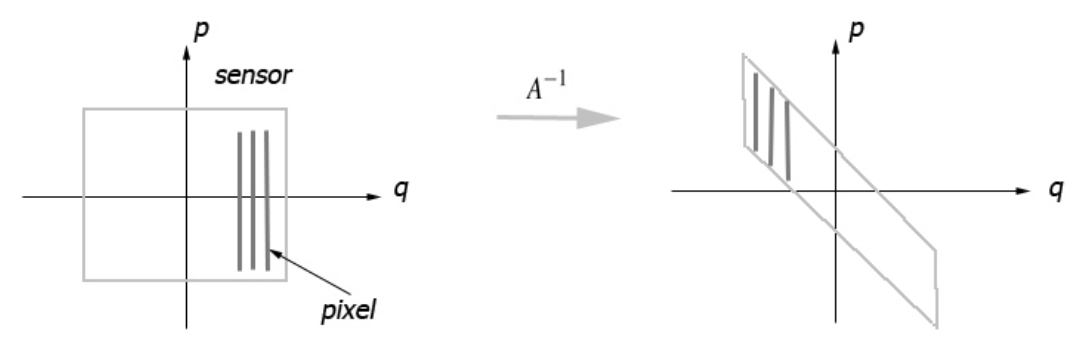

Fig. 3. Sampling pattern of one microlens in Plenoptic 2.0 camera.

Regarding camera design, the spatio-angular tradeoff for the Plenoptic 2.0 camera is not fixed by the number of microlenses. Rather, the spatio-angular tradeoffs are determined by the optical geometry ( $a$ and $b$ ). To counter edge effects, relatively large microlenses can be used.

\section{Conclusion}

Our analysis compares the two plenoptic cameras and shows the reason for the increased spatial resolution in Plenoptic 2.0. The resolution is not only decoupled from the number of microlenses, but it also has great flexibility determined by the $a / b$ ratio. Considering that the light field of most natural surfaces has Lambertian redundancy, Plenoptic 2.0 sampling is capable of making a better spatioangular tradeoff.

\section{References}

1. T. Adelson and J. Wang. Single lens stereo with a plenoptic camera. IEEE Transactions on Pattern Analysis and Machine Intelligence, pages 99-106, 1992.

2. K. Fife, A. El Gamal, and H.-S. P. Wong. A 3Mpixel multi-aperture image sensor with $0.7 \mu \mathrm{m}$ pixels in $0.11 \mu \mathrm{m}$ CMOS. In IEEE ISSCC Digest of Technical Papers, pages 48-49, February 2008.

3. Marc Levoy and Pat Hanrahan. Light field rendering. ACM Transactions on Graphics, pages 31-42, 1996.

4. G Lippmann. Epreuves reversibles donnant la sensation du relief. Journal of Physics, 7(4):821-825, 1908.

5. Andrew Lumsdaine and Todor Georgiev. Full resolution lightfield rendering. Technical report, Adobe Systems, January 2008.

6. Andrew Lumsdaine and Todor Georgiev. The focused plenoptic camera. In IEEE International Conference on Computational Photography (ICCP), 2009.

7. Ren Ng. Digital light field photography. PhD thesis, Stanford University, Stanford, CA, USA, 2006. Adviser-Patrick Hanrahan. 